\title{
(2) OPEN ACCESS \\ Impact of the COVID-19 pandemic on emergency medical service response to out-of-hospital cardiac arrests in Taiwan: a retrospective observational study
}

\author{
Jiun-Hao Yu, ${ }^{1,2}$ Chien-Yu Liu, ${ }^{2,3}$ Wei-Kung Chen, ${ }^{2,3}$ Shao-Hua Yu, ${ }^{2,3}$ Fen-Wei Huang, ${ }^{3}$ \\ Ming-Tzu Yang, ${ }^{3}$ Chih-Yu Chen, ${ }^{4}$ Hong-Mo Shih (1) 2,3,5
}

\begin{abstract}
Handling editor Caroline Leech

Additional supplemental material is published online only. To view, please visit the journal online (http://dx.doi. org/10.1136/emermed-2020210409).
\end{abstract}

${ }^{1}$ Department of Emergency Medicine, China Medica University Hospital, Hsinchu, Taiwan

${ }^{2}$ College of Medicine, China Medical University, Taichung, Taiwan

${ }^{3}$ Department of Emergency Medicine, China Medical University Hospital, Taichung, Taiwan

${ }^{4}$ Department of Industrial Engineering and Enterprise Information, Tunghai University, Taichung, Taiwan

${ }^{5}$ Department of Public Health, China Medical University,

Taichung, Taiwan

\section{Correspondence to}

Dr Hong-Mo Shih, College of medicine, China Medical University, Taichung 404, Taiwan;

homoe042002@hotmail.com

J-HY and C-YL contributed equally.

J-HY and C-YL are joint first authors.

Received 16 July 2020 Accepted 5 July 2021

Published Online First 14 July 2021

\section{Check for updates}

(C) Author(s) (or their employer(s)) 2021. Re-use permitted under CC BY-NC. No commercial re-use. See rights and permissions. Published by BMJ.

To cite: Yu J-H, Liu C-Y, Chen W-K, et al. Emerg Med 2021;38:679-684.

\section{ABSTRACT \\ Background Emergency medical service (EMS)} personnel have high COVID-19 risk during resuscitation. The resuscitation protocol for patients with outof-hospital cardiac arrest (OHCA) was modified in response to the COVID-19 pandemic. However, how the adjustments in the EMS system affected patients with OHCA remains unclear.

Methods We analysed data from the Taichung OHCA registry system. We compared OHCA outcomes and rescue records for 622 cases during the COVID-19 outbreak period (1 February to 30 April 2020) with those recorded for 570 cases during the same period in 2019. Results The two periods did not differ significantly with respect to patient age, patient sex, the presence of witnesses or OHCA location. Bystander cardiopulmonary resuscitation and defibrillation with automated external defibrillators were more common in 2020 (52.81\% vs $65.76 \%, p<0.001 \%$, and $23.51 \%$ vs $31.67 \%$, $p=0.001$, respectively). The EMS response time was longer during the COVID-19 pandemic $(445.8 \pm 210.2 \mathrm{~s}$ in 2020 vs $389.7 \pm 201.8 \mathrm{~s}$ in $2019, \mathrm{p}<0.001)$. The rate of prehospital return of spontaneous circulation was lower in 2020 (6.49\% vs 2.57\%, $p=0.001) ; 2019$ and 2020 had similar rates of survival discharge $(5.96 \%$ vs $4.98 \%$ ). However, significantly fewer cases had favourable neurological function in 2020 (4.21\% vs $2.09 \%, p=0.035)$.

Conclusion EMS response time for patients with OHCA was prolonged during the COVID-19 pandemic. Early advanced life support by EMS personnel remains crucial for patients with OHCA.

\section{INTRODUCTION}

Front-line healthcare workers are at high risk when working with patients with COVID-19. Recent research data indicated that although nosocomial infection was not an critical transmission factor in COVID-19, healthcare workers accounted for $15 \%-18 \%$ and in some cases even as high as $20 \%$ of the infected COVID-19 population. ${ }^{1}$ As a result of early intervention to mitigate the spread of COVID19, Taiwan was one of the few countries without a lockdown or healthcare crisis in early $2020 .^{2}$ Despite considerable efforts devoted to preventing the spread of COVID-19, the first case in Taiwan was diagnosed on 21 January $2020 .^{3}$

Emergency medical service (EMS) personnel are particularly exposed to COVID-19 risk when resuscitating patients with out-of-hospital cardiac

\section{Key messages}

What is already known on this subject

- The prehospital resuscitation protocol of emergency medical service had been modified during the COVID-19 pandemic.

- Emergency medical service response time is associated with the survival outcomes of patients with out-of-hospital cardiac arrest (OHCA).

\section{What this study adds}

- In this analysis of Taichung OHCA registry system, during the COVID-19 pandemic, there was an increase in ambulance response time from call to departure, and emergency medical technicians spent longer at scene performing resuscitation.

- Although the percentages of witnessed collapse, bystander cardiopulmonary resuscitation and defibrillation were higher during the pandemic, the survival outcomes from OHCA were worse than in the prior year.

arrest (OHCA). This is because resuscitation entails numerous aerosol-generating procedures, including chest compressions, airway management and positive pressure ventilation. Both the American Heart Association and Taiwan Society of Emergency Medicine had published pandemic-adapted guidance for basic and advanced life support. ${ }^{45}$ In particular, dispatchers were instructed to screen all calls to identify COVID-19 symptoms, travel history and prior contact with those suspected to have COVID19. ${ }^{4}$ Emergency medical technicians (EMTs) should also wear personal protective equipment (PPE) before entering the scene. ${ }^{4}$ Ventilation strategies with lower aerosolisation risk had been applied to patients with OHCA in prehospital settings during the early stages of the COVID-19 outbreak. $^{6}$

In Taiwan from 2000 to 2012, the 180-day survival rate for patients with OHCA was approximately 9.8\%. ${ }^{7}$ Early EMS activation, bystander cardiopulmonary resuscitation (CPR), high-quality $\mathrm{CPR}$ and early defibrillation can improve the survival rates. ${ }^{8}$ Due to advances in resuscitation, the global survival rate of patients with OHCA who receive CPR had been increasing since the 1970s (8.6\% in 1976-1999 vs 9.9\% in 2010-2019). ${ }^{9}$ 
This study aimed to examine the effect of Taiwan's modified OHCA protocol on the outcomes of patients with OHCA treated by the EMS during the COVID-19 pandemic in an urban city. We obtained a city-based EMS registry data set for before and during the COVID-19 pandemic and analysed the differences in out-of-hospital management and patient with OHCA outcomes between the two periods.

\section{MATERIALS AND METHODS \\ Study background and population}

Taichung city, located in Central Taiwan, has an area of $2215 \mathrm{~km}^{2}$. Of Taiwan's 20 cities, Taichung is the second most populous, with approximately 2.8 million people. The first case of COVID-19 infection in Taiwan was identified on 21 January 2020. As of 30 April 2020, approximately 63716 suspected cases had been tested for COVID-19, of which 429 cases were confirmed to be infected, including 374 imported cases and 55 locally transmitted cases, and resulting in six deaths; furthermore, 140000 people had been quarantined. Approximately 40 confirmed COVID-19 cases were located in Taichung. ${ }^{3}$ A reproduction number of 0.06 was reported for all imported cases. ${ }^{10}$

To evaluate the impact of Taiwan's modified COVID-19 OHCA protocol on patient outcomes, we analysed data obtained from the Taichung OHCA registration system. We enrolled all patients with OHCA between 1 February and 30 April 2020. For comparison, we included all patients with OHCA during the same period in 2019. Patients for whom resuscitation was not attempted, those aged younger than 20 years and those with OHCA as a result of trauma were excluded from the cohort. This study was approved by the Institutional Review Board of China Medical University (CMUH108-REC3-068).

\section{Prehospital resuscitation and registry system for OHCA in Taichung}

In Taichung, there are more than 2500 EMS-attended OHCA cases each year. ${ }^{11}$ For each OHCA case, the dispatch centre provides guidance in dispatcher-assisted CPR for members of the public and sends two to four EMTs for rescue. In Taichung, prehospital management of OHCA is generally undertaken by EMTs trained in basic life support (BLS) and consists of chest compressions and the use of an automated external defibrillator (AED) and oxygenation with a bag valve mask or laryngeal mask airway (LMA). If Emergency Medical Technician Paramedics (EMT-P) personnel are present at the scene, they can provide endotracheal intubation and administer epinephrine intravenously. However, because of the small number of EMT-P and no offline medical direction, advanced life support, including intubation and epinephrine, is rarely performed in prehospital resuscitation in Taichung. Regarding local customs and legal issues, EMS personnel rarely terminate resuscitation at the scene unless requested by family members.

EMT attendance records and resuscitation records for all patients with OHCA were recorded by the EMT in charge using an Utstein-style template. Information on patient outcome after resuscitation was provided by the hospital in charge. Since 1997 , such information has been collected by the quality management team of the Taichung Dispatch Center and integrated into the Taichung OHCA registry system. ${ }^{12}$

\section{Modified rescue protocol during the COVID-19 outbreak}

After the first case of COVID-19 was announced in Taiwan on 21 January 2020, ${ }^{13}$ for all 1-1-9 calls in Taichung, dispatchers were required to obtain information on travel history, occupation and prior contact with those with high COVID-19 risk. To reduce the spread of COVID-19, EMTs worked in isolated units of two. For patients assessed by dispatchers as having high COVID-19 risk and those with uncertain COVID-19 history, EMTs were instructed to wear PPE: an N95 mask, a fluid-resistant gown, two layers of disposable gloves, shoe covers, a protective face shield and a pair of goggles. For patients assessed by dispatchers as having a low risk of COVID-19, EMTs were advised to wear a surgical mask, a pair of disposable gloves, a protective face shield and a pair of goggles. EMTs were also required to reassess the risk of COVID-19 when they arrived at the scene. If in any doubt, they had to upgrade their PPE level without hesitation. According to the Taiwan Society of Emergency Medicine recommendation, AED use and hand-only CPR were an alternative for patients with OHCA during the COVID-19 pandemic. ${ }^{4}$ To minimise aerosol generation, the airway should be managed either with LMA in conjunction with a high-efficiency particulate air filter or a non-rebreathing mask at the lowest flow. Details about conventional and additional management for patients with OHCA before and during the COVID-19 pandemic in Taichung are presented in online supplemental figure S1.

\section{Data collection}

Demographical characteristics were compared between the two cohorts. The demographical characteristics included on the Utstein-style template were age, sex, OHCA location, presence of witnesses, bystander CPR and AED use status. EMS response time, defined as the time interval from call to EMT arrival at the scene, was also compared between the two study periods. ${ }^{14}$ The differences in rescue duration between 2019 and 2020 were analysed in detail. These specific durations comprised the intervals from call to EMT departure, from EMT departure to arrival at the scene, from arrival at the scene to leaving the scene and from leaving the scene to reaching the hospital.

\section{Outcome measurement}

The outcome measurements followed the recommended core data recorded on the Utstein-style template. The primary outcome was the percentage of survival with favourable neurological function at hospital discharge. Patient outcomes after resuscitation, including prehospital return of spontaneous circulation (ROSC), sustained ROSC and survival to hospital discharge, were also compared. Prehospital ROSC was defined as any ROSC that had been achieved before arrival at the receiving hospital. Sustained ROSC was defined as maintaining spontaneous circulation for at least 2 hours, including patients with prehospital ROSC. Neurological function was measured at hospital discharge. In accordance with the 5-point Cerebral Performance Category (CPC) Scale, ${ }^{15}$ neurological status was classified as follows: good recovery (CPC 1), moderate disability (CPC 2), severe disability (CPC 3), persistent vegetative state (CPC 4) and brain death (CPC 5). CPC 1 and 2 were considered favourable neurological outcomes, whereas CPC 3-5 and mortality were considered unfavourable outcomes. ${ }^{16}$ According to Taiwan's epidemic prevention policy, all high-risk patients should be tested for COVID-19 through nasopharyngeal swab specimens by reverse transcriptase PCR. The results of the COVID-19 tests would be reported to the Health Bureau of Taichung City Government. If the COVID-19 test results were positive, all exposed healthcare providers, including EMTs, would be notified, and self-health monitoring for at least 14 days would be required. 


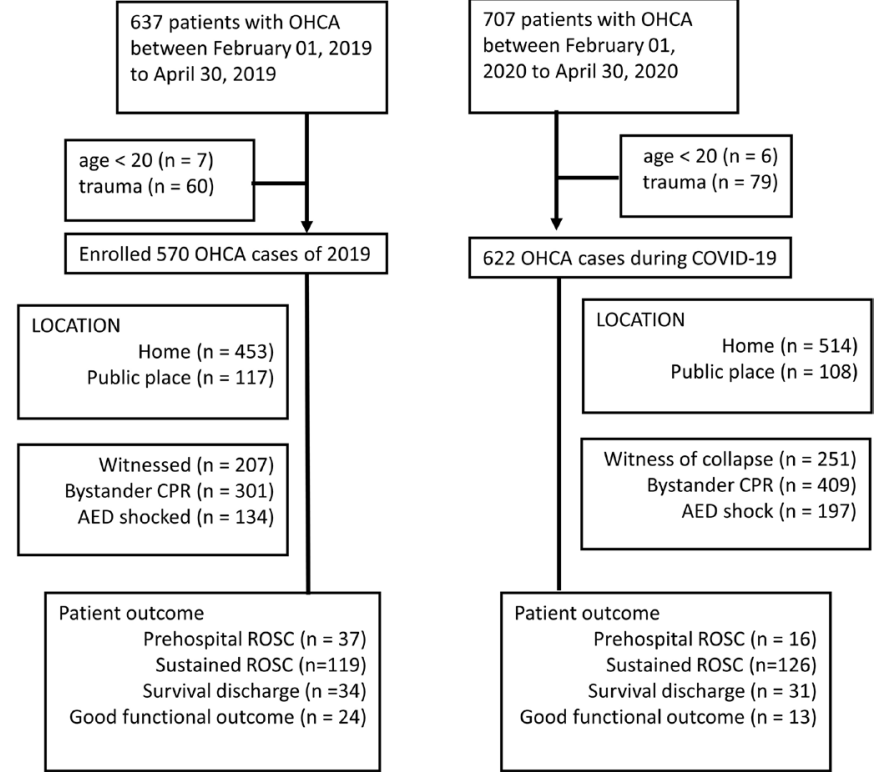

Figure 1 Patient enrolment and demographical characteristics using the Utstein-style template. OHCA, out-of-hospital cardiac arrest; CPR, cardiopulmonary resuscitation; ROSC, return of spontaneous circulation; $A E D$, automated external defibrillator.

\section{Statistical analysis}

We used SAS V.9.4 for all statistical analyses. Continuous variables are presented as mean \pm SD and were compared using the independent sample $t$ test. Categorical variables were analysed using a $\chi^{2}$ test and are presented as number and proportion. All assessments were two-sided, and a p value of $<0.05$ was deemed as statistically significant.

\section{RESULTS}

In total, 707 cases of OHCA were identified between 1 February and 30 April 2020. Six cases were excluded because the patient was younger than 20 years, and 79 were excluded because the patient had traumatic OHCA, resulting in 622 eligible cases for the 2020 group. Similarly, 570 OHCA cases were identified from the registry for 1 February to 30 April (2019) as 2019 comparison group (figure 1). Although not all patients with OHCA were tested for COVID-19, there were no confirmed COVID-19 cases among patients with OHCA in Taichung City during the 2020 study period.

Age and sex did not differ significantly between the two groups. More collapses were discovered at home, and more witnessed collapses were noted in 2020 than in 2019, but the difference was not statistically significant. The overall percentage of cases receiving bystander CPR was higher in 2020 (52.81\% vs $65.76 \%, \mathrm{p}<0.001)$. Bystander CPR in public places was similar between the two periods, whereas bystander CPR at home was increased by $13.5 \%$ in 2020 study period. The percentage of cases where an AED was used was higher in the 2020 group $(23.51 \%$ vs $31.67 \%, p=0.001)$. Because EMTs worked in isolated units of two, the proportion of OHCA cases attended by two EMTs was obviously higher in the 2020 group $(69 \%$ vs $81 \%, \mathrm{p}<0.001)$. Table 1 lists the demographical characteristics and prehospital management of patients with OHCA before and during the COVID-19 pandemic.

There was less than a minute difference in the average EMS response time to the OHCA between the two time periods. The mean EMS response time to an OHCA was 56 s longer in the 2020
Table 1 Demographical data and prehospital management of patients with out-of-hospital cardiac arrest before and during the COVID-19 pandemic

\begin{tabular}{|c|c|c|c|}
\hline Variables & $\begin{array}{l}2019 \\
(n=570)\end{array}$ & $\begin{array}{l}2020 \\
(n=622)\end{array}$ & $P$ value \\
\hline Age, mean & $70.93 \pm 16.45$ & $70.41 \pm 16.21$ & 0.585 \\
\hline Male gender & $353(61.93)$ & $394(63.34)$ & 0.614 \\
\hline Location & & & 0.163 \\
\hline Home & $453(79.47)$ & $514(82.64)$ & \\
\hline Others & $117(20.53)$ & $108(17.36)$ & \\
\hline Witness of collapse & $207(36.32)$ & $251(40.35)$ & 0.152 \\
\hline \multicolumn{4}{|l|}{ Bystander CPR } \\
\hline Overall & $301(52.81)$ & 409 (65.76) & $<0.001$ \\
\hline Public place $(n=225)$ & $65 / 117(55.56)$ & $72 / 108(66.67)$ & 0.088 \\
\hline Home $(n=967)$ & $236 / 453(52.10)$ & $337 / 514(65.56)$ & $<0.001$ \\
\hline $\begin{array}{l}\text { Automated external } \\
\text { defibrillator shock }\end{array}$ & $134(23.51)$ & $197(31.67)$ & 0.001 \\
\hline $\begin{array}{l}\text { EMT response time } \\
\text { (seconds) }\end{array}$ & $389.7 \pm 201.8$ & $445.8 \pm 210.2$ & $<0.001$ \\
\hline Numbers of rescuer & & & $<0.001$ \\
\hline 2 & $393(68.95)$ & $504(81.03)$ & \\
\hline 3 & $173(30.35)$ & $115(18.49)$ & \\
\hline 4 & $4(0.70)$ & $3(0.48)$ & \\
\hline $\begin{array}{l}\text { Airway and oxygen } \\
\text { management }\end{array}$ & & & 0.253 \\
\hline Supraglottic airway & 499 (87.54) & $537(86.33)$ & \\
\hline Bag valve mask & $67(11.75)$ & $74(11.90)$ & \\
\hline Non-rebreathing mask & $4(0.70)$ & $11(1.77)$ & \\
\hline
\end{tabular}

CPR, cardiopulmonary resuscitation; EMT, emergency medical technician.

group ( $389.7 \pm 201.8 \mathrm{~s}$ vs $445.8 \pm 210.2 \mathrm{~s}, \mathrm{p}<0.001)$. The interval from call to EMT departure increased $39.8 \mathrm{~s}(38.3 \pm 49.35 \mathrm{~s}$ vs $78.1 \pm 69.01 \mathrm{~s}, \mathrm{p}<0.001)$ in the 2020 group. The transfer times were similar between the two periods. However, in the 2020 group, EMTs spent longer time at the scene, which indicates the time required to perform resuscitation in the field was longer in the 2020 group $(675.1 \pm 245.5 \mathrm{~s}$ vs $756.1 \pm 289.7 \mathrm{~s}, \mathrm{p}<0.001)$. Figure 2 shows the detailed time difference of the prehospital course between the 2019 group and the 2020 group.

The percentage of cases that achieved prehospital ROSC was lower during the 2020 study period than it was during the 2019 comparison period $(6.49 \%$ vs $2.57 \%, p=0.001)$. The 2019 and 2020 groups had similar rates of sustained ROSC and survival at hospital discharge. However, significantly fewer cases had favourable neurological function at discharge in the 2020 group (4.21\% vs $2.09 \%, \mathrm{p}=0.035$; table 2 ).

In figure 3, we combined all OHCA cases, including the 2020 pandemic group and 2019 comparison group, and presented all reported outcomes of OHCA cases percentage of total cases) by EMS response time (minutes). The probability of prehospital ROSC was $4.86 \%$ when the EMS response time was less than 5 min but only $1.88 \%$ when the response time exceeded 10 min. Similarly, the likelihoods of achieving sustained ROSC, survival to hospital discharge and survival with favourable neurological function were all superior if the response time was less than $5 \mathrm{~min}$.

\section{DISCUSSION}

In this study, we found an increased EMS response time during the COVID-19 pandemic compared with the same time period in the previous year, particularly for the interval from call to 


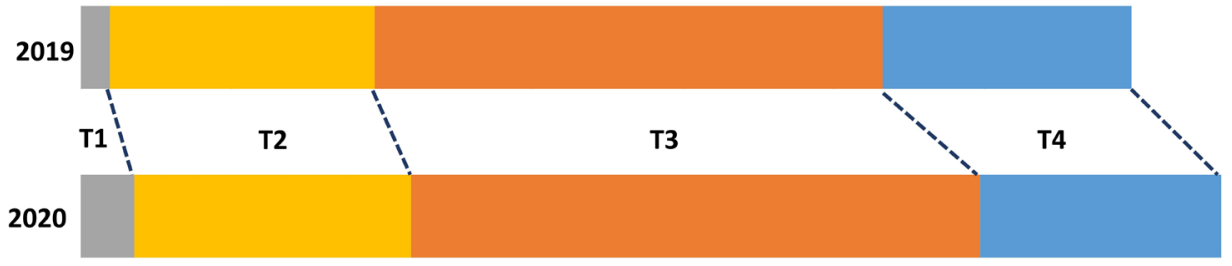

\begin{tabular}{|c|c|c|c|}
\hline EMT rescue timing & $\begin{array}{l}2019 \\
\text { (sec) }\end{array}$ & $\begin{array}{l}2020 \\
\text { (sec) }\end{array}$ & $P$ value \\
\hline T1 (Interval between call to EMT departure) & $38.3 \pm 49.35$ & $78.1 \pm 69.01$ & $<0.001$ \\
\hline T2 (Interval between departure to arrival at scene) & $351.8 \pm 194.1$ & $367.7 \pm 195.8$ & 0.161 \\
\hline T3 (Interval between arrival and leave the scene) & $675.1 \pm 245.5$ & $756.1 \pm 289.7$ & $<0.001$ \\
\hline T4 (Interval of transportation to hospital) & $330.5 \pm 239.1$ & $321.5 \pm 216.2$ & 0.501 \\
\hline
\end{tabular}

Figure 2 Emergency medical technician (EMT) rescue durations for out-of-hospital cardiac arrest before and during the COVID-19 pandemic.

EMT departure. EMTs also remained at the scene longer. Compared with the same period in 2019, an increased proportion of two-EMT rescue was observed in 2020. Although the percentages of bystander CPR and defibrillation using AED were higher, the short-term and longer-term survival outcomes after OHCA worsened in the 2020 pandemic group; the percentages of patients with prehospital ROSC and those who survived with favourable neurological function were both lower during the 2020 pandemic period.

Although the percentages of witnessed collapse, bystander CPR and defibrillation using AED were higher in 2020 than in 2019, the prehospital ROSC and survival with favourable neurological function rates were not higher. In the Lombardy region of Italy, ${ }^{17}$ an area that saw a severe COVID-19 outbreak, the median EMS arrival time was 3 min longer in 2020 than it was in 2019. Among patients resuscitated by the EMS, the percentage of prehospital ROSC in 2020 was $11.7 \%$ lower than that in 2019. Studies have revealed that a shorter EMS response time is associated with more favourable outcome for patients with OHCA. ${ }^{14}$ Response time also has a significant independent effect on survival rate, regardless of bystander CPR. ${ }^{18} \mathrm{~A}$ recent study found that EMS arrival times longer than 10 min reduce the chance of survival to hospital admission. ${ }^{19}$ A shorter response time enables EMTs to provide high-quality CPR earlier, manage the airway earlier and perform defibrillation earlier, which are crucial elements in the so-called chain of survival that is taught in advanced life support courses. ${ }^{8}$ In the current study, we also found that longer EMS response time was associated with poorer survival outcomes after OHCA (figure 3 and online supplemental table S1). Therefore, maintaining the EMS response time within the average range is critical during the COVID-19 pandemic.

EMS response time had increased by approximately $1 \mathrm{~min}$ during the 2020 pandemic period, which was similar with the report from Paris region. ${ }^{20}$ The increase in EMS response time during the COVID-19 pandemic was driven largely by the

\begin{tabular}{|c|c|c|c|}
\hline Patient outcomes & $\begin{array}{l}2019 \\
(n=570)\end{array}$ & $\begin{array}{l}2020 \\
(n=622)\end{array}$ & $P$ value \\
\hline Prehospital ROSC & 37 (6.49) & $16(2.57)$ & 0.001 \\
\hline Sustained ROSC & $119(20.88)$ & $126(20.26)$ & 0.791 \\
\hline Survival discharge & $34(5.96)$ & $31(4.98)$ & 0.456 \\
\hline Favourable neurological function & $24(4.21)$ & $13(2.09)$ & 0.035 \\
\hline
\end{tabular}

ROSC, return of spontaneous circulation. increase in the time between the dispatchers receiving the call and the EMT crew departing. The dispatchers spent additional time gathering information regarding COVID-19 symptoms, travel history and contact history with individuals at high risk of COVID-19. ${ }^{4}$ During the COVID-19 pandemic, EMTs worked in isolated units of two, and the volume of incoming calls also increased. ${ }^{21}$ Consequently, dispatchers required more time to dispatch EMTs for rescue. EMTs were instructed to wear PPE during the pandemic period, and these additional preparations require from at least a few seconds to a few minutes. ${ }^{4} 22$ The current study found that the aforementioned adjustments, although necessary during the COVID-19 pandemic, led to delayed EMS response.

EMTs spent longer performing resuscitation in the field during the COVID-19 outbreak in 2020 than the same period in 2019. EMTs were required to reassess the risk of COVID-19 when they arrived at a scene. The use of PPE might interfere with the way EMTs perform resuscitation for patients with OHCA. Moreover, EMTs had to take additional measures to minimise aerosol generation during resuscitation (online supplemental figure S1). To prevent the spread of COVID-19 between EMS staff, twoEMT attendance for rescue was encouraged. However, reduced numbers of EMT personnel might result in increased duration of resuscitation at the scene and poorer survival outcomes among patients with OHCA. ${ }^{23}{ }^{24}$ In this study, we found more frequent two-EMT attendance for rescues during the COVID-19 pandemic in 2020. Consistent with previous studies, longer on-scene resuscitation times and poorer survival outcomes were observed during the 2020 COVID-19 pandemic period in this study.

In addition, the overall percentage of cases in which bystander performed CPR was higher in 2020, especially in cases of cardiac arrest at home. Although the overall outcomes of OHCA cases were also poorer, the percentage of cases with receipt of bystander CPR has declined in other pandemic-hit areas. ${ }^{25}$ Several reasons may exist for this disparity. First, there has been no community-level COVID-19 pandemic in Taiwan, which may be why laypersons are willing to provide CPR. Second, although Taiwan has not implemented a lockdown policy, the government recommends that people reduce social activities and avoid going to public places as much as possible. This probably underlies the observed increase in cases of collapse occurring in homes and that of cases reported as 'witnessed collapses' in the study period, compared with the previous year. People may be more willing to perform CPR on someone they are close to. During the 2020 study period, because of more cardiac arrests occurring 


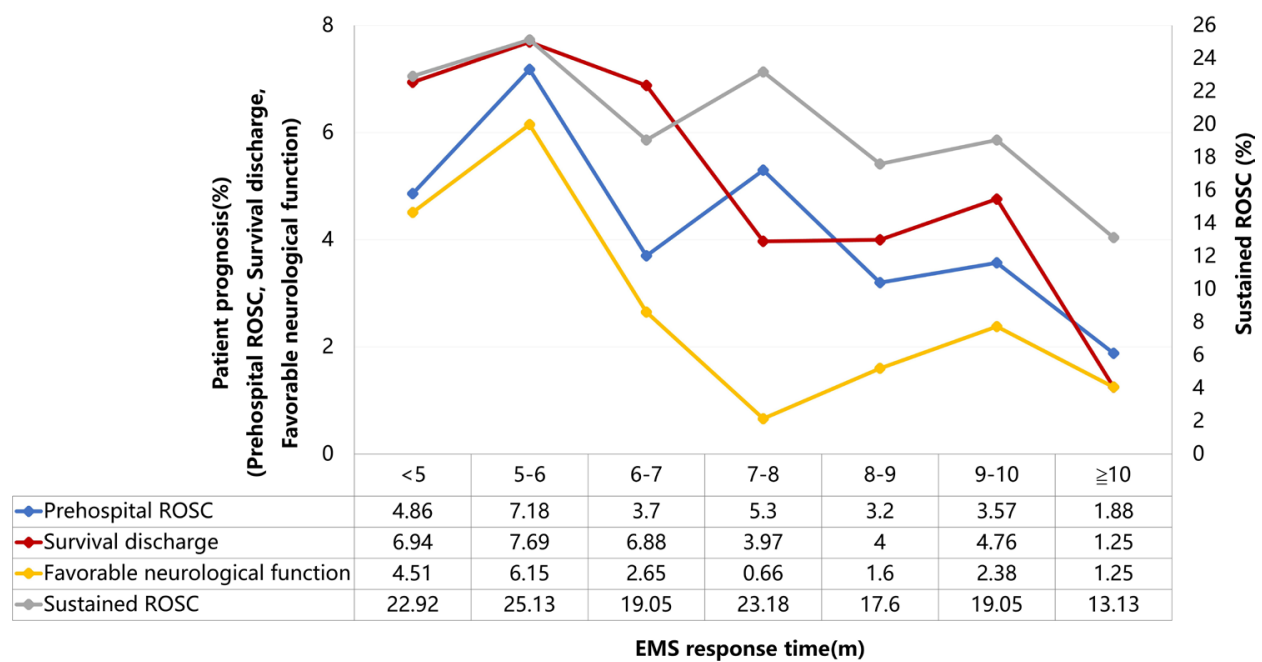

Figure 3 All reported outcomes of out-of-hospital cardiac arrest cases percentage of total cases) by emergency medical service (EMS) response time (minutes). ROSC, return of spontaneous circulation.

in homes, the percentage of cases that received bystander CPR might have increased accordingly. Finally, due to the promotion of dispatcher-assisted CPR in past years, the proportion of bystander CPR increased from $35.72 \%$ in 2016 to $54.46 \%$ in $2019 .^{11}$ This may be another reason why the percentage of cases receiving bystander CPR was higher in 2020. Patients who were witnessed collapses and received bystander CPR were found to be more likely to have shockable rhythms. ${ }^{26}$ Similar to the higher percentages of witnessed collapse and bystander CPR, more cases with shockable rhythms were also recognised and defibrillated using AEDs during the 2020 study period. A recent report by Sultanian et al also indicated that during the 2020 pandemic, a higher proportion of cardiac arrests occurring at home, a higher rate of witnessed arrests, and more defibrillations were delivered by bystanders. ${ }^{27}$ The chance of discharged alive had also decreased during the 2020 pandemic. The societal impact of the COVID-19 pandemic may affect the characteristics and outcomes of patients with OHCA, which were also found in our study.

In Taichung, most patients with OHCA had their spontaneous circulation restored after being transported to the receiving hospital. EMTs mainly provided BLS for patients with OHCA. The EMS response time and transportation interval to the hospital were relatively shorter in Taichung than other regions. Furthermore, EMTs rarely terminated the resuscitation unless requested by family members. These may be the reasons why the percentage of prehospital ROSC was much lower than that of in-hospital sustained ROSC, which was also observed in a previous study in Taichung. ${ }^{28}$ These factors can also explain why the percentages of sustained ROSC and survival to discharge were similar between 2019 and 2020 despite a longer EMS response time in 2020. Despite no difference existing in sustained ROSC, the longer EMS response time in the 2020 study period resulted in lower percentages of prehospital ROSC and survival with favourable neurological function.

\section{Limitations}

The strength of this study is its use of a database of an EMS system during the COVID-19 pandemic. However, the study has some limitations. First, these were regional data, and Taiwan controlled the COVID-19 pandemic relatively well. Therefore, the overall results may not be applicable to other regions. Second, the COVID-19 epidemic in Taiwan was controlled relatively well in a timely manner, and furthermore, the operation of the entire EMS system was also maintained favourably. The impact of EMS response on patients with OHCA may vary between regional epidemic situations. However, the adjustment of the prehospital resuscitation protocol for patients with OHCA is consistent with the universal recommendation. Therefore, our results are still representative. Third, the study period was relatively short, and more time may be required to observe the long-term impact of the COVID-19 pandemic on the EMS system. Fourth, the time from collapse to calling EMS and the quality of bystander CPR, which might affect the prognosis after OHCA, could not be measured in the Taichung OHCA registry. Finally, in accordance with the Personal Data Protection Act, personal information about high or low COVID-19 risk has been delinked from the registry; thus, we were unable to perform a subgroup analysis of patients at high and low risk of COVID-19. Nevertheless, because a city-based registry database was employed, the analysis results are still credible and can reflect the actual impact of COVID-19.

\section{CONCLUSION}

The EMS system's response time was prolonged during the COVID-19 pandemic, which may have negatively affected OHCA survival outcomes. Early advanced life support provided by EMS personnel remains crucial for favourable survival outcomes of patients with OHCA.

Acknowledgements We would like to thank all the EMS personnel and Fire Bureau of Taichung City Government. We also thank all researcher of the Critical Care Research Team of emergency department of China Medical University Hospital, Taichung: Wei-Kung Chen, MD; Hong-Mo Shih; Tai-Yi Hsu, MD; Shao-Hua Yu, MD; Jiun-Hao Yu, MD; Chien-Yu Liu, MD; Chin-Han Lin, MD; Chih-Yu Chen, MD; Yu-Hsin Chang, MD; Fen-Wei Huang, MSc; Ming-Tzu Yang, MS; and Shih-Yun Emma Wu, MD.

Contributors J-HY: Conceptualisation and writing —original draft. C-YL: Writingoriginal draft-and resources. W-KC: Funding acquisition and supervision. SHY: Software and validation. F-WH: Formal analysis and visualisation. M-TY: Data curation. C-YC: Resources. H-MS: Methodology, writing —review and editing-and investigation.

Funding This work was supported, in part, by China Medical University Hospital under grant (DMR-109-057 and DMR-109-058). 
Disclaimer The funding agencies had no role in the design and conduct of the study; collection, management, analysis and interpretation of data; preparation, review and approval of manuscript; or decision to submit manuscript for publication.

Competing interests None declared.

Patient and public involvement statement Patients or the public were not involved in the design, conduct, reporting or dissemination plans of our research.

Patient consent for publication Not required.

Ethics approval The Institutional Review Board of China Medical University approved this observational study (CMUH107-REC1-059).

Provenance and peer review Not commissioned; externally peer reviewed.

Data availability statement Data are available on reasonable request. Raw data were generated at Taichung OHCA registry system of Fire Bureau of Taichung City Government. Derived data supporting the findings of this study are available from the corresponding author (H-MS) on request.

Supplemental material This content has been supplied by the author(s). It has not been vetted by BMJ Publishing Group Limited (BMJ) and may not have been peer-reviewed. Any opinions or recommendations discussed are solely those of the author(s) and are not endorsed by BMJ. BMJ disclaims all liability and responsibility arising from any reliance placed on the content. Where the content includes any translated material, BMJ does not warrant the accuracy and reliability of the translations (including but not limited to local regulations, clinical guidelines, terminology, drug names and drug dosages), and is not responsible for any error and/or omissions arising from translation and adaptation or otherwise.

Open access This is an open access article distributed in accordance with the Creative Commons Attribution Non Commercial (CC BY-NC 4.0) license, which permits others to distribute, remix, adapt, build upon this work non-commercially, and license their derivative works on different terms, provided the original work is properly cited, appropriate credit is given, any changes made indicated, and the use is non-commercial. See: http://creativecommons.org/licenses/by-nc/4.0/.

ORCID iD

Hong-Mo Shih http://orcid.org/0000-0001-7732-3594

\section{REFERENCES}

1 Ali S, Noreen S, Farooq I, et al. Risk assessment of healthcare workers at the frontline against COVID-19. Pak J Med Sci 2020;36(COVID19-S4):S99-103.

2 Lin C, Braund WE, Auerbach J, et al. Policy decisions and use of information technology to fight COVID-19, Taiwan. Emerg Infect Dis 2020;26:1506-12.

3 Taiwan Centers for Disease Control. The statistics of COVID-19 in Taiwan. Available: https://www.cdc.gov.tw/En [Accessed 1 Oct 2020].

4 Recommendations for pre-hospital respiratory treatment during the COVID-19 epidemic. Taiwan Emergency Medicine Bulletin;3:e2020030213 https://www.sem. org.tw/EJournal/Detail/200

5 Edelson DP, Sasson C, Chan PS, et al. Interim guidance for basic and advanced life support in adults, children, and neonates with suspected or confirmed COVID-19: from the emergency cardiovascular care Committee and get with the GuidelinesResuscitation adult and pediatric task forces of the American heart association. Circulation 2020;141:e933-43.

6 Yang W-S, Hou S-W, Lee B-C, et al. Taipei Azalea - Supraglottic airways (SGA) preassembled with high-efficiency particulate air (HEPA) filters to simplify prehospital airway management for patients with out-of-hospital cardiac arrests (OHCA) during coronavirus disease 2019 (COVID-19) pandemic. Resuscitation 2020;151:3-5.
7 Wang $\mathrm{C}-\mathrm{Y}$, Wang J-Y, Teng N-C, et al. The secular trends in the incidence rate and outcomes of out-of-hospital cardiac arrest in Taiwan--a nationwide population-based study. PLoS One 2015;10:e0122675.

8 Link MS, Berkow LC, Kudenchuk PJ, et al. Part 7: adult advanced cardiovascular life support: 2015 American heart association guidelines update for cardiopulmonary resuscitation and emergency cardiovascular care. Circulation 2015;132:\$444-64.

9 Yan S, Gan Y, Jiang N, et al. The global survival rate among adult out-of-hospital cardiac arrest patients who received cardiopulmonary resuscitation: a systematic review and meta-analysis. Crit Care 2020;24:61.

10 Liu J-Y, Chen T-J, Hwang S-J. Analysis of imported cases of COVID-19 in Taiwan: a nationwide study. Int J Environ Res Public Health 2020;17. doi:10.3390/ ijerph17093311. [Epub ahead of print: 0905 2020].

11 Fire Bureau of Taichung City Goverment. 2019 annual statistical report of Taichung City fire bureau. Available: https://www.fire.taichung.gov.tw/form/index-1.asp? Parser $=2,10,74,53, \ldots 506$; page 110 [Accessed 1 Oct 2020].

12 Huang SK, Chen C-Y, Shih H-M, et al. Dispatcher-assisted cardiopulmonary resuscitation: differential effects of landline, mobile, and transferred calls. Resuscitation 2020;146:96-102.

13 Cheng S-C, Chang Y-C, Fan Chiang Y-L, et al. First case of coronavirus disease 2019 (COVID-19) pneumonia in Taiwan. J Formos Med Assoc 2020;119:747-51.

14 Pell JP, Sirel JM, Marsden AK, et al. Effect of reducing ambulance response times on deaths from out of hospital cardiac arrest: cohort study. BMJ 2001;322:1385-8.

15 Jennett B, Bond M. Assessment of outcome after severe brain damage. Lancet 1975;1:480-4

16 Edgren E, Hedstrand U, Kelsey S, et al. Assessment of neurological prognosis in comatose survivors of cardiac arrest. BRCT I study Group. Lancet 1994;343:1055-9.

17 Baldi E, Sechi GM, Mare C, et al. Out-Of-Hospital cardiac arrest during the Covid-19 outbreak in Italy. N Engl J Med 2020;383:496-8.

18 Bürger A, Wnent J, Bohn A, et al. The effect of ambulance response time on survival following out-of-hospital cardiac arrest. Dtsch Arztebl Int 2018;115:541-8.

19 Baldi E, Caputo ML, Savastano S, et al. An Utstein-based model score to predict survival to hospital admission: the UB-ROSC score. Int I Cardiol 2020;308:84-9.

20 Marijon E, Karam N, Jost D, et al. Out-Of-Hospital cardiac arrest during the COVID-19 pandemic in Paris, France: a population-based, observational study. Lancet Public Health 2020:5:e437-43.

21 Dami F, Berthoz V. Lausanne medical dispatch centre's response to COVID-19. Scand I Trauma Resusc Emerg Med 2020;28:37.

22 Ortega R, Gonzalez M, Nozari A, et al. Personal protective equipment and Covid-19. N Engl I Med 2020

23 Nishi T, Maeda T, Takase K, et al. Does the number of rescuers affect the survival rate from out-of-hospital cardiac arrests? two or more rescuers are not always better than one. Resuscitation 2013;84:154-61.

24 Tsai BM, Sun J-T, Hsieh M-J, et al. Optimal paramedic numbers in resuscitation of patients with out-of-hospital cardiac arrest: a randomized controlled study in a simulation setting. PLoS One 2020;15:e0235315.

25 Uy-Evanado A, Chugh HS, Sargsyan A, et al. Out-Of-Hospital cardiac arrest response and outcomes during the COVID-19 pandemic. JACC Clin Electrophysiol 2021;7:6-11.

26 Song J, Guo W, Lu X, et al. The effect of bystander cardiopulmonary resuscitation on the survival of out-of-hospital cardiac arrests: a systematic review and meta-analysis. Scand I Trauma Resusc Emerg Med 2018;26:86.

27 Sultanian P, Lundgren P, Strömsöe A, et al. Cardiac arrest in COVID-19: characteristics and outcomes of in- and out-of-hospital cardiac arrest. A report from the Swedish Registry for cardiopulmonary resuscitation. Eur Heart J 2021:42:1094-106.

28 Chen Y-C, Yu S-H, Chen W-J, et al. Dispatcher-Assisted cardiopulmonary resuscitation: disparity between urban and rural areas. Emerg Med Int 2020;2020:1-7. 